\title{
Status of Chironomids (Diptera) as Potential Bioindicators
}

\author{
Deepak Rawal $^{1}$, Gyata Prajapat ${ }^{2}$ and Himani Verma ${ }^{3}$ \\ ${ }^{1,2,3}$ Department of Zoology, Mohanlal Sukhadia University, \\ Udaipur, Rajasthan 313001, India
}

\begin{abstract}
Chironomids are one of the most dominant, widespread and diverse aquatic invertebrate taxa in freshwater systems. Chironomids are closely related to mosquitoes (Culicidae) and biting midges (Ceratopogonidae). Chironomids are important contributors of carbon and energy flow to higher trophic levels. Bioindicators gives us an idea of the health of an ecosystem. Some organisms are very sensitive to pollution in their environment, so if pollutants are present the organism may change its morphology, physiology or behavior or it could even die. Structures of polytene chromosomes, such as the BRs and NOR, may be used as biomarkers for indication of heavy metal pollution. Aberrations (inversions, duplications, deletions, deficiencies etc.) in polytene chromosomes of Chironomids accords with environmental pollution. Chironomids show different response to stress induced by toxic agents.
\end{abstract}

Keywords: Chironomids, bioindicators, polytene chromosomes, mentum, pollution.

\section{Introduction}

Chironomids are members of the family Chironomidae (Diptera). Chironomids often called true flies, non biting midges and blind mosquitoes. Chironomids are one of the most dominant, widespread and diverse aquatic invertebrate taxa in freshwater systems. Chironomids are closely related to mosquitoes (Culicidae) and biting midges (Ceratopogonidae) (Armitage et al, 1995). Approximately 5000 species of Chironomids reported worldwide (Ferrington et al, 2008). Their life cycle includes three aquatic development stages (egg, larva and pupa) and a terrestrial reproductive stage (Winged adult). Chironomids spend most of their lives as benthic larvae living and feeding on the sediment (Oliver, 1971). The larval stage has four instars, and may last from less than two weeks to several years depending on species and environmental conditions. Many larval Chironomids are red because of hemoglobin analog in their hemolymph, which allows these organisms to tolerate low oxygen concentrations in the water and another aspect is the possibility that this pigment is able to metabolize xenobiotics. Chironomids are an important food source for large predatory invertebrates, fishes, and birds (Hudson et al, 1990). Chironomids have an important role in aquatic food webs, representing a major link between producers, such as phytoplankton and benthic algae and secondary consumers. Chironomids are important contributors of carbon and energy flow to higher trophic levels. They are infamous because of their small size, they are not vector of any disease, they are taxonomically difficult to identify and they do not have any aesthetic value (Singh \& Rawal, 2016).

Bioindicators are any species or group of species whose function, population or status can reveal the qualitative status of the environment. Bioindicators gives us an idea of the health of an ecosystem. Some organisms are very sensitive to pollution in their environment, so if pollutants are present the organism may change its morphology, physiology or behavior or it could even die. The level of pollution of continental waters as a result of industrial, routine, and agricultural human activity increases. Environmental pollution, including water sources, is a serious problem facing plants, animals and human beings. Habitat destruction, pollution, and climate changes due to anthropogenic impacts are the main causes of loss of biodiversity, mainly in streams and rivers. Although these threats are increasing, the effects of these disturbances regarding the impacts of many pollutants on freshwater biodiversity are still poorly understood. Freshwater invertebrate fauna plays key ecological roles in the maintenance of aquatic ecosystem services. Mainly in secondary production and energy flow dynamics. Among these organisms, aquatic insects are the most important, because they are the major proportion of functional feeding groups helping in ecosystem dynamics. Furthermore, they have a noteworthy importance to freshwater monitoring, protection, and 
conservation due to their sensitivity to respond to unfavorable changes in environmental conditions (Barbosa et al, 2001). Some invertebrates are used for better estimation of environmental pollution both on the individual and population levels and on the community level. Among biological indicators, Chironomids are distinguished because they make up the most widespread insect family and they have received attention by researchers worldwide due to their outstanding abilities as biological indicators of environmental conditions (Pinder, 1986).

\section{Results and Discussion}

\subsection{Morphological deformities in Chironomid} larvae as a Tool:

Larvae of Chironomids live at the bottom, where pollution products often accumulate as a result of human activity. Generally, eutrophic water is inhabited by species eating organic meals and bottom deposits with the filtration method. These deposits are used by larvae when they grasp feed and build dwelling, as a result, larvae are constantly affected by polluting agents. However, larvae are not only affected by pollutants but also accumulate them. The morphological deformities of Chironomids larvae offer useful way of estimating sediment toxicity. Midge larvae are able to metabolize organic contaminants, but the breakdown product may also be responsible for morphological abnormalities. The mentum and epipharyngis deformities incidence was highly correlated with contaminant and a decrease in $\mathrm{pH}$ and dissolved oxygen. They are directly involved in the transformations that contaminants can undergo in freshwater ecosystem. They accumulate various pollutants in their tissue and play a role as a primary link in the food chain leading from sediment to fish, birds and humans. The larvae of some Chironomids species have been used as bioindicators of the contamination of sediments. Deformities in the antennae also provide excellent early warning signals for the detection of toxic pollutants in the environment (Warwick, 1990). The total deformities correlated closely with deformities of mentum. Lenat (1993) categorized the deformities of mentum into three classes:

Class I: includes slight deformities which are difficult to separate from the "chipped" teeth.

Class II: includes more conspicuous deformities, such as extra teeth, missing teeth, large gaps, and distinct asymmetry.

Class III: includes the larvae that suffer severe deformation, including at least two class II characters.

\subsection{Aberrations in polytene chromosomes of Chironomid larvae as a Tool:}

The somatic cells of Chironomids are very sensitive to the influence of environmental contaminants. Balbiani rings system (BR) and nucleolar organizer (NOR) were considered to be interesting models for studying the response of the genome to different environmental stress agents (Hudson \& Ciborowski, 1996). The most relevant cytogenetic responses to the genotoxication of pollutants consist in functional and structural changes of the salivary gland polytene chromosomes. These functional changes can be traced mainly by variations in a number of indicator such as, a decrease in the puffing activity of the Balbiani rings (BRs) and the Nucleolar Organizer Region (NOR), the appearance of novel puffs, the decondensation of centromeres and telomeres as well as asynapses of both homologues. Balbiani rings correspond to genes coding the highmolecular-weight silk proteins that are necessary for the production of the tubes where the larvae live. The Balbiani rings are generally sites of intensive transcription, which can be detected by their characteristic large puffs. Decreases in the level of transcription were observed after treatment with different concentrations of cyclohexamide, galactose and ethanol. The NOR is the genomic region, where ribosomal RNA genes are concentrated and are essential for cellular maintenance. NORs are sites of high transcription (Hudson \& Ciborowski, 1996). The level of transcription is related to variations in the protein requirements of the cells. In the majority of cells of salivary gland, the NOR occurs at the highest activity during the larval stage. The structure chromosome aberrations that are the most common and easiest to observe consist of inversion, deletions, deficiencies and amplifications. They can be used to assess the genotoxic effect of stress agent in the environment and can be considered to be the best marker of trace-material genotoxicity (Michailova et $a l, 2011)$.

Certain heavy metals ( $\mathrm{Cu}, \mathrm{Zn}, \mathrm{Co}, \mathrm{Fe}, \mathrm{Mg}$ etc.) are required as trace elements for normal cellular functions. Their presence in trace amounts is considered important for living organisms, because they represent active sites for number of enzymes which are involved in oxidation-reduction reactions. However, when physiological concentration exceeds, heavy metals are toxic to cells. Effect of heavy metals can be deleterious when they are released in water either in high concentrations for short periods, causing acute toxicity, or in low concentration but for long periods, causing chronic toxicity and leading disorder in development, growth, maturation, reproduction, hatching, which not necessarily result in early death. 
Therefore some models Chironomid are used for tracing the effects of some heavy metals on the genome structure and functional organization. The biological approaches for detecting and assessing the impact of contaminants on ecosystems have a number of advantages. Chironomids provide direct means of studying the impact of contaminants in the environment. Chironomids allow improving evaluation of ecological contaminants at individual, population and community level. In acute and chronic tests for hazard identification, Chironomids can be maintained in controlled conditions and therefore their response to specific toxic agents has a high level of repeatability. The higher concentrations of trace metals $(\mathrm{Cd}, \mathrm{Zn}, \mathrm{Pb}$, and $\mathrm{Cr}$ ) affect the genome of cytogenetically studied Chironomids. Genome instability of Chironomids was manifested by two ways: 1. Fixed chromosome rearrangements; homozygous inversions and tandem fusions created new gene linkage groups and show intensive micro evolutionary process of Chironomids. 2. Somatic structural (inversion, deficiencies, deletions, breaks) and functional alterations (decreasing the activity of the key structures: Balbiani rings (BRs) and nucleolar organizer (NOR) in salivary gland chromosomes of cytogenetically studied Chironomids (Kiknadze et al, 2010). Detecting somatic rearrangements in salivary gland chromosomes of these widely distributed aquatic insect is potentially one of the best validated bioassay and can be used as a cost effective early warning signals of environmental damage in freshwater system.

\section{Conclusions}

The frequency of mouth parts deformities in Chironomids correlate with environmental pollution. Polytene chromosomes of Chironomids can also be used as potential bioindicators of environment quality. Structures of polytene chromosomes, such as the BRs and NOR, may be used as biomarkers for indication of heavy metal pollution. Aberrations (inversions, duplications, deletions, deficiencies etc.) in polytene chromosomes of Chironomids accords with environmental pollution. Chironomids show different response to stress induced by toxic agents. Overall Chironomid larvae has the good potential for providing early warnings of adverse long term effects of toxic agents at the individual, population and community level and can be used as cost effective tool for indicating environmental health of aquatic ecosystem.

\section{References}

[1] Armitage PD, Cranston PS \& Pinder LCV. The Chironomidae: Biology and ecology of non-biting midges. 1st ed., Chapman and Hall, pp.1-538, (1995).
ISSN 2455-6378

[2] Barbosa F, Galden $\mathrm{N}$ and Callisto $\mathrm{M}$. Biodiversity assessment of benthic macroinvertebrates in altitudinal ecosystem of Serra do Cipo (MG Brazil). Brazilian Journal of Biology, 61(2): 239-248, (2001).

[3] Ferrington LC, Coffman WP \& Berg MB. Chironomidae: An introduction to the aquatic insects of North America. Merritt RW, Cummins KW, Berg MB (Eds). Kendall and Hunt, pp. 847-989, (2008).

[4] Hudson LA \& Cibrowski JH. Spatial and taxonomic variation incidence of mouth parts deformities in midge larvae (Diptera: Chironomidae: Chironomini). Can. J. Fish. Aquat. Sci., 53: 297-304, (1996).

[5] Hudson PL, Lenat, DR, Caldwell, BA \& Smith D. Chironomidae of the Southeastern United States: A checklist of species and notes on biology, distribution, and habitat. Fish and Wildlife Research, 7 (4): 1-4, (1990).

[6] Kiknadze II, Istomina AG, Wulker WF and Vallenduuk HJ. The karyotype of Chironomus uliginosus Keyl. Becmhuk BOTUC, 14 (1): 22-28, (2010).

[7] Lenat DR. Using mentum deformities of Chironomus larvae to evaluate the effects of toxicity and organic loading in streams. Journal of the North American Benthological Society, 12 (3), 265-269, (1993).

[8] Michailova P, Petrova N, Sella G, Bovero S, Ramella L, Regoli F \& Zelano V.Genotoxic effects of chromium on polytene chromosomes of Chironomus riparius Meigen (Diptera: Chironomidae). Caryologia 54 (1): 59-71, (2001).

[9] Michailova P, Szarek-Gwiazda E, Kownacki A \& Warchalowska-Sliwa E. Biodiversity of Chironomidae (Diptera) and genome response to trace metals in the environment. Pesticides 1-4: 41-48, (2011).

[10] Oliver DR. Life history of the Chironomidae. Annual Review of Entomology, 16, 211-30, (1971).

[11]Pinder LCV. Biology of freshwater Chironomidae. Ann. Rev. Ent., 31: 1-23, (1986).

[12] Singh P \& Rawal D. Biodiversity of Chironomids from Udaipur region (India). International Journal of Advanced Research, 4(9): 502-505, (2016).

[13] Warwick WF. The use of Morphological Deformities in Chironomid Larvae (Diptera: Chironomidae) for Biological Effects Monitoring. Environ. Can. IWD Scientific Series No. 173, NHRI Paper No. 43:1-34, (1990). 